\title{
Modeling Monitoring of Age Dependent Diseases as a Reflection of Aging Genes Expressing
}

\author{
S. Kulishov, Member, IACSIT, and O. Iakovenko
}

\begin{abstract}
The purpose of this work is solving problem of age dependent diseases monitoring as a reflection of aging genes expressing using system and anti-system comparison, graphic modeling, programming, exploratory analysis on the principles of Cantor, von Koch sets, Cantor square fractal and anti-fractal concepts. Proposed and tested an algorithm of fractal and anti-fractal concepts for exploratory analysis of age dependent diseases manifestations, as a reflection of genes expressing. Our algorithm will help maximize insight, uncover underlying structure, extract important variables, develop models and determine optimal factor settings, improve quality of diagnosis and treatment, body and genome nets building.
\end{abstract}

Index Terms - Modeling, diseases, genes.

\section{PRerequisites for ANALysis AND Modeling OF AgING DYNAMICS}

Ageing is one of the most important and relevant topics of all mankind. In the last years there has been an intensive search for candidates for the role of genes of death and longevity in humans [1]. In view of the large number of candidate genes involved in aging, is now the focus is on the genes that are homologous to genes determining longevity in animals of other species, and genes responsible for the development of the main diseases associated with aging. Establishing genetic features longevity and determination of the genetic status of key genes involved in the pathogenesis of age-dependent diseases, are the main approaches to the identification of the key components of active aging and longevity.

Candidate genes participate in the processes of aging can be classified as:

- Involving in maintaining the balance of cell tissues

(apoptosis genes, telomerase);

- Control the integrity of the genome and DNA repair;

- Determine resistance to stress (heat shock genes, and antioxidant protection);

- Cause epigenetic changes (methylation, carbonylation and nitrosylation).

Recently, it was found that oxidative stress (excessive formation of reactive oxygen species - ROS) can significantly accelerate the shortening of telomeres and is an integral part of cellular aging, as well as exacerbating pathologies characteristic of old age. To neutralize ROS in

Manuscript received November 5, 2012; revised January 24, 2013.

S. Kulishov is with department of Internal Medicine No. 1, HSEIU "Ukrainian Medical Stomatological Academy", Poltava, Ukraine (e-mail: study@umsa.com.ua, kulishov@meta.ua ).

O. Iakovenko is with endocrinology separation of daily permanent establishment. V. P. Komissarenko Institute of Endocrinology and Metabolism AMS Ukraine (e-mail: admin@iem.kiev.ua). the cell there is a system of antioxidant protection. Repression or overexpression of antioxidant enzyme genes significantly alters life expectancy. One of the key enzymes of this system is superoxide dismutase (MnSOD, SOD2) [2]. At various model organisms MnSOD was demonstrated influence on aging and longevity. There are data on the association of gene polymorphism SOD2 (SOD2-9T/C) with the development of pathologies seniors: atherosclerosis, hypertension, cardiomyopathy, diabetic nephropathy, some cancers, etc [3].

Anti-aging medicine is a medical specialty founded on the application of advanced scientific and medical technologies for the early detection, prevention, treatment, and reversal of age-related dysfunction, disorders, and diseases. It is a healthcare model promoting innovative science and research to prolong the healthy lifespan in humans. As such, anti-aging medicine is based on principles of sound and responsible medical care that are consistent with those applied in other preventive health specialties.

Medical science has cataloged many signs of senescence [1]. It manifests as dozens of changes in cells, tissues, and organs during aging. Human life is supported by a complex network of biochemical substances and reactions which affect the physical state and vitality of the body and mind. Senescent changes can be seen in the rate and outcome of many of these reactions [1]. However, many of these changes are secondary effects of senescence, rather than primary causes. Chronic inflammation is a well-known corollary of the aging process and is believed to significantly contribute to morbidity and mortality of many age-associated chronic diseases [2]. However, the mechanisms that cause age-associated inflammatory changes are not well understood. Particularly, the contribution of cell stress responses to age-associated inflammation in 'non-inflammatory' cells remains poorly defined [2].

Chronic inflammation associated with the aging process has been implicated in a host of degenerative disease states including osteoarthritis, atherosclerosis, type-2 diabetes. Age-associated chronic inflammatory states are distinct from inflammation triggered by infection. It is presently unclear to what extent chronic inflammatory states in older individuals represent autoimmune processes caused by deregulation of the immune system [2]. Alternatively, these states may arise as a consequence of an increased cell stress response in old cells triggered by molecular damage incurred over a lifetime.

We consider that analysis of age dependent diseases manifestations, as a reflection of genes expressing, help us to determine rhythms of aging and antiaging process, its possible mechanisms. Monitoring of age dependent diseases manifestations by sensors of motion, registration of 
electrocardiograms (ECGs), electromyograms (EMGs), electro-encephalograms (EEGs), respiratory rhythm, glycemic level may be basis for this analysis.

It's known that the emerging m-Health concept represents the evolution of e-health systems loosely defined as the use of the Internet for healthcare to mobile applications without guaranteed Internet connectivity [3]. The advances in $\mathrm{m}$-Health systems are driven by the developments in wireless communications, pervasive, and wearable technologies [3]. A typical Wireless Body Area Network (WBAN) consists of a number of inexpensive, lightweight, and miniature sensor platforms, each featuring one or more physiological sensors, e.g. motion sensors, ECGs, EMGs, EEGs [3]. The sensors could be located on the body as tiny intelligent patches, integrated into clothing, or implanted below the skin or muscles [3].

The purpose of this work is solving problem of age dependent diseases monitoring as a reflection of aging genes expressing using system and anti-system comparison, graphic modeling, programming, exploratory analysis on the principles of Cantor, von Koch sets, Cantor square fractal and anti-fractal concepts.

\section{The Methodology of Analysis And Modeling AgE Diseases MONITORING AS A REFLECTION OF AGING GENES EXPRESSING}

Algorithm of system and anti-system comparison for solving clinical problems of age dependent diseases monitoring as a reflection of aging genes expressing boils down to: the formation of the pro-aging and anti-aging factors set, Formulation sub-thesis and sub-antithesis, which are key in solving creative task and its blocking; formulation of preliminary solution of this task; Grouping constituent elements into system and anti-system complexes, the compositions; Formulation of oxymorons from system and anti-system complexes as sub-themes;

Unification of oxymorons as sub-themes in the general oxymoron theme, solution through fractal structures, focusing- defocusing for overcoming of limiting search of central theme the transformation of intermediate preliminary solution in the final decisions.

The methodologies of fractal data transformation for exploratory statistical analysis have 3 steps [4]. The first step is:

- Selection of informative numerical dependent variabilities for transformation to categorical types by using Cantor, von Koch or others sets principles as basis, triggers for exploratory clinical research analysis.

The second step is statistical analysis of these informative numerical variabilities:

- Determination of mean, standard error of mean, standard deviation, 95\% confidence interval for mean, median, minimum, maximum, range, quartiles, interquartile range, skewness, kurtosis.

- Determination of the variabilities distribution parametric or nonparametric by single-factor the Kolmogorov-Smirnov test; Shapiro-Wilk W test and graphical methods: frequency distribution histograms stem \& leaf plots; scatter plots; box \& whisker plots; normal probability plots: PP and QQ plots; graphs with error bars (Graphs: Error Bar).

The third step is:

- To choose using Cantor or von Koch sets principles for transformation informative numerical variabilities to categorical types by results of statistical analysis. More high level of standard deviation, interquartile range is better for using Cantor or von Koch sets principles.

Algorithm of exploratory data analysis using the technology of the iteration process as for receiving Cantor set [4], [5]:

- Selection of informative numerical dependent variabilities;

- Transformation these informative numerical dependent variabilities to categorical variabilities;

- Formation categorical variabilities in the form of subgroups with the maximum, median and minimum values;

- Formation of a new categorical variabilities in the form of sub-subgroups with maximum, median and minimum values in the subgroups with the highest and lowest values (closed ring);

- Formation categorical variabilities in the form of subgroups as a result of an iterative process as for Cantor set;

- Statistical analysis of the data;

- Determination of the variabilities distribution parametric or nonparametric by single-factor the Kolmogorov-Smirnov test; Shapiro-Wilk W test and graphical methods: frequency distribution histograms stem \& leaf plots; scatter plots; box \& whisker plots; normal probability plots: PP and QQ plots; graphs with error bars (Graphs: Error Bar);

- Transformations that may be normalize of non-normality data: If residuals have a right skew, should apply a square-, a cube- or fourth-root, a logarithmic, and an inverse transformation to data. If residuals have a left skew, should raise to the second, third or fourth power, an exponential transformation to data;

- ANOVA - Analysis of Variance, with variations depending on the linear nature of variability. Method of multiple comparison groups Tukey HSD, Scheffe, Bonferroni if deviations were homogeneous for the test Levene, and in the absence of homogeneity - the criteria Tamhane's T2, Games-Howell;

- Nonparametric equivalent of ANOVA / MANOVA Kruskal-Wallis test;

- Formulation of a conclusion based on statistical analysis.

- The methodologies of other fractal and anti-fractal data transformation for exploratory statistical analysis have basis of different iterative process.

\section{Summary Data of AnAlysis And Modeling AgE Diseases MONITORING AS A REFLECTION OF AGING GENES EXPRESSING}

Algorithm of rhythms and fractals aging and anti-aging diagnosis is reduced to the determination of the episodes of maximum and minimum or without manifestations of age-dependent pathology. Age-dependent pathology, as the 
expression of genes of aging, is presented by the symptoms, syndromes, diseases, multimorbidity states, more often the cardiovascular, respiratory, endocrine, digestive and nervous systems. Triggers of the aging process are the effects of anti-aging factors consumption. Monitoring the activity of internal organs allows to evaluate the dynamics of these processes.

Algorithm for diagnosis of aging rhythms and fractals is reduced to the determination the episodes of:

- Small range of diurnal changes of the heart rate (chronotropic insufficiency);

- The appearance of disturbances of rhythm and conduction from minimum to maximum, life dangerous level;

- Arterial hypertensive or hypotensive reactivity, increasing arterial pulse pressure;

- Hypopnea or apnea during the night;

- Syndrome "restless legs" at night;

- Hypoglycemia, hyperglycemia;

- Desynchronosis of cardiovascular, respiratory, glycemia, hormonal rhythms.

The mechanisms that favor the appearance of aging fractals on the gene, the subcellular (including the mitochondrial level), cellular, tissue, organ, system, organism as a whole are:

- Syndromes of consumption of aging progradient and antigradient, pro-morbidity and anti-morbidity, pro-oxidant and anti-oxidant, pro-inflammatory and anti-inflammatory, pro-ishemic and anti-ischemic, pro-arrhythmic and anti-arrhythmic, and other factors;

- Changes of the cardiovascular, brain, skeletal muscle electromagnetic processes in space and time, as Mobius strip;

- Changes of cardiovascular, respiratory, glycemia, hormonal rhythms similar to those known in the climate effects, as El Nino and La Nina;

- The transition from mono- to multimorbidity;

- Narrowing of chaotic influences that lead to aging and anti-aging disbalance.

Examples of the results of the algorithm for using principles of Cantor set for Exploratory Data Analysis of clinical research data are presented by determination of the dependence between tolerance to stress, inflammation syndrome, coronary and myocardial failure, arterial pressure, ventricular arrhythmias in the patients with coronary heart disease and essential hypertension [6]-[11].

Fractal analysis of monitoring glycemic profile and arterial pressure helped with diagnosis of the sum negative effects in the patients with 1st diabetes mellitus in combination with essential hypertension [12]. Daily changes in glucose levels, blood pressure, heart rate are the basis of individual diagnosis of pathogenic mechanisms, the correction of the treatment of the patients with diabetes mellitus in combination with essential hypertension [12].

Additional studies may clarify the mechanisms of aging and the role of [1]:

- Oxidation, glycation, cross-linking, and other chemical modifications all act to impair the molecular functioning of multiple vital components, including DNA, membranes, the extracellular matrix (ECM), enzymes, and structural proteins. Modifications which accumulate faster than they are repaired or recycled will cause progressive deterioration over time;

- Junk molecules and aggregates accumulate inside and outside of cells;

- The redox potential poise of some cells changes in response to these chemical modifications. This results in altered gene expression, altered enzyme activity, and altered signaling pathways;

- Repair and recycling mechanisms slow down;

- A minority of deteriorating cells release chemicals which harm other, healthy cells;

- The neuro-endocrine and immune systems seem to follow a developmental program of decline, which may cause them to send chemical signals of differentiation and death to various tissues;

- Cells are lost by apoptosis and necrosis, especially among nondividing cells of the heart, skeletal muscle, and brain substantia nigra. Organs and tissues deteriorate over time when cells are lost faster than they are replaced;

- Stem cells stop dividing and no longer replace essential cells or regenerate tissues.

New perspective additional studies may clarify the mechanisms of aging and the role of the sinus node as the primary pacemaker of the heart [13]. A complex interplay of heterogeneities is assumed to be the basic mechanism that the sinus node can drive the heart. This interplay can be disturbed by e.g. diseases, drugs or mutations. In this work the effect of a mutation on the sinus node function were investigated. Algorithm of anti-aging rhythms and fractals is reduced to the determination of various episodes of synchronization of rhythms in the range of the golden section, Fibonacci numbers, the lack of activation of the above signs of age-dependent pathology. Increasing of duration of the age-dependent pathology deactivation determines adaptation to aging and reflects decreased expression of genes of aging. Therefore, measurement data of wild-type and mutant I Kr channels were integrated with aid of optimization procedures into the heterogeneous sinus node model of Zhang et al. [13]. The measurement data shows a shift of the steady-state inacti-vation to more positive potentials. Simulated central sinus node cells lose their ability to depolarize spontaneously. Peripheral cell are also effected by the mutation. The main changes are the shortening of the action potential duration from $108 \mathrm{~ms}$ to $84 \mathrm{~ms}$ and the increase of auto-rhythmic frequency from $6.37 \mathrm{~Hz}$ to $7.62 \mathrm{~Hz}$ due to an increased mean I $\mathrm{Kr}$ current. In a future study the bradycardial effect of this mutation will be shown in a tissue model.

This algorithm is a system of operations to convert data in the result as a generalized solution.

The presence of efficient algorithms for solving problems which are before, contributes to a positive outcome.

Thus, the proposed technology presented as a graphical model and program by languages "Dragon" [14], promotes understanding of complex principles of medical and biological problems, improve the quality of solving monitoring age dependent diseases as reflection of aging 
genes expressing problem.

“Dragon” [14] (Fig. 1).

Results of modeling age dependent diseases on language

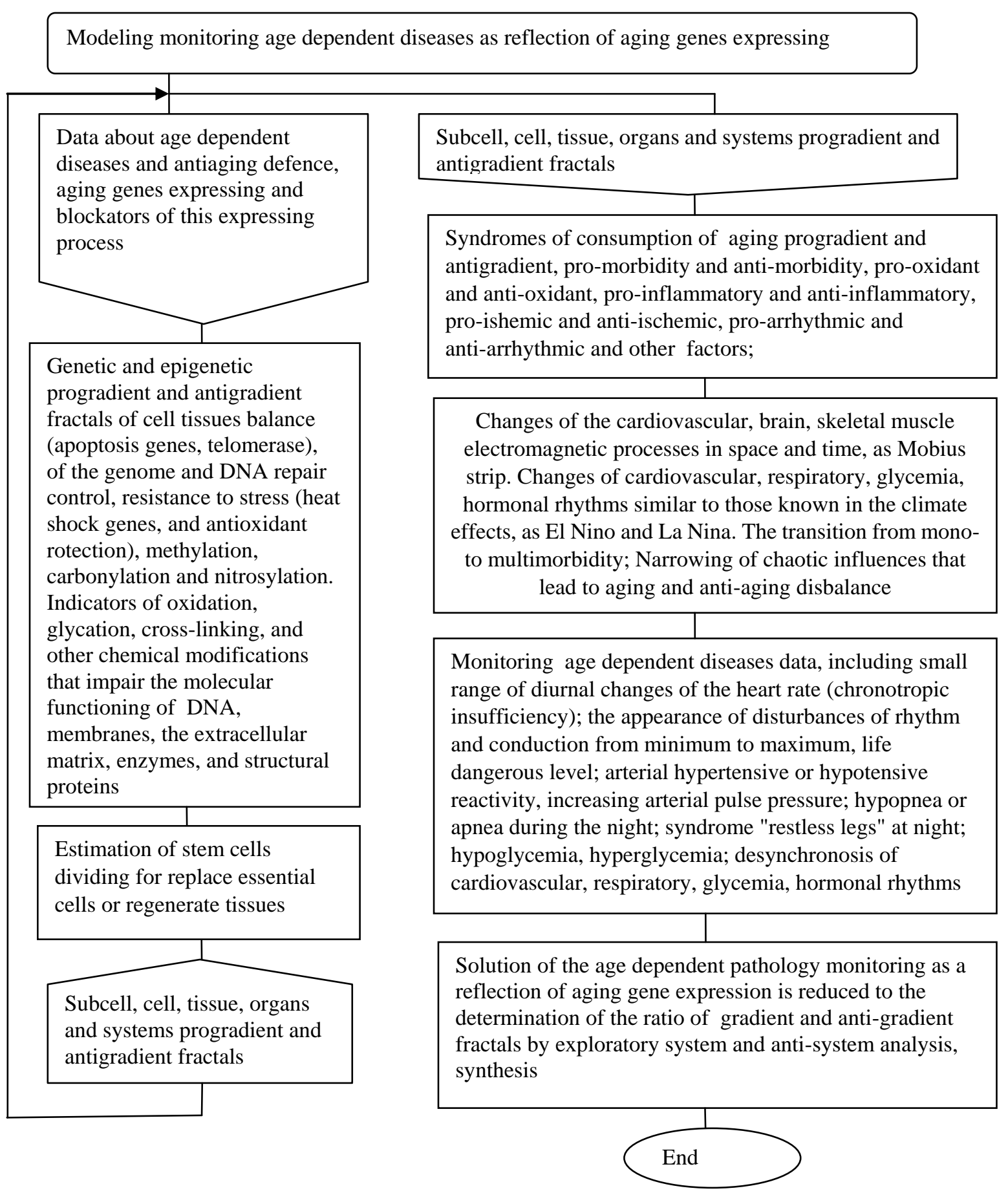

Fig. 1. Modeling monitoring age dependent diseases as reflection of aging genes expressing

\section{CONCLUSION}

Our algorithm system and anti-system comparison, graphic modeling, programming, exploratory analysis on the principles of Cantor, von Koch sets, Cantor square fractal and anti-fractal concepts will help maximize insight, uncover underlying structure, extract important variables, develop models and determine optimal factor settings, may improve possibilities of diagnosis and treatment.

Solution of the age dependent pathology monitoring as a reflection of aging gene expression is reduced to the determination of the ratio of gradient and anti-gradient fractals by exploratory system and anti-system analysis, synthesis.

Proposed algorithm is a system of operations to convert data in the result as a generalized solution.

Implementation of method of estimation aging "quants"-fractals will promote to resolve antiaging treatment tasks.

\section{REFERENCES}

[1] J. Vijg, "Aging of the genome: the dual role of dna in life and death," New York, USA: Oxford University Press Inc., pp. 384, 2007. 
[2] A. Kriete "Biomarkers of aging: combinatorial or system model?" Sci Aging Knowledge Environ, pe1, 2006.

[3] E. Jovanov "Wireless technology and system integration in body area networks for m-health applications," in Proceedings of the 27th Annual International Conference of the IEEE Engineering in Medicine and Biology Society, 2005.

[4] S. K. Kulishov and O. M. Iakovenko, "Fractals as triggers exploratory statistical analysis of clinical pharmacological data," International journal of pharmacology and pharmaceutical technology (IJPPT), vol. 1, Issue 1, pp. 53-57, 2012.

[5] C. Shaver, "An exploration of the cantor set," Rockhurst University .09, James and Elizabeth Monahan Summer Research Fellowship, Summer 2008, MT4960: Mathematics Seminar, Spring, pp. 19, 2009.

[6] V. A. Bobrov and S. K. Kulishov, "The adaptive ischemic and reperfusion syndromes in the patients with ischemic heart disease: mechanisms, diagnosis, substantiation of therapy," Poltava: Dyvosvit, 2004, pp. 240.

[7] S. K. Kulishov and O. M. Iakovenko, "Solving clinical problems using system and anti-system comparison, graphic modeling," Innovative Medicine and Biology, Canadian International Monthly Reviewed Journal (CIJIMB), no. 3, pp. 30-42, 2011.

[8] S. Kulishov and I. Kudria "Stress and inflammation as determinative factors in the patients with coronary heart disease and essential hypertension," Final programme\& Abstract Book of the European Conference, IInd edition "Heart, Vessels \& Diabetes", nos. 3-5, Athens, Greece, pp. 50, 2011.

[9] S. Kulishov, K. Vakulenko, and I. Latoha, "Myocardial electrical instability as the derivative of inflammation consumption of antiiflammatory factors syndrome, changes in geometry depolarization of atria, ventricles in the patients with coronary heart disease," Absract book of "Rhythm 2011," Congress, Marseile, France, pp. 39, 2011.

[10] S. K. Kulishov, I. P. Kudria, and K. Ye. Vakulenko, "Method of diagnosis of circadian stress and stress-limitative rhythms in patients with ischemic heart disease in combination with essential hypertension," The patent of Ukraine for utility model no. 41598 IPC (2009) A 61 B 5/02. - Bulletin no. 10, 2009.

[11] S. K. Kulishov and K. Ye. Vakulenko, "Method of diagnosis of systemic disturbances of desynchronosis of arterial pressure, respiratory, cardiac rhythms," The patent of Ukraine for utility model no. 41598 IPC (2009) A 61 B 5/02; A 61 B 5/0205. - Bulletin no. 12 , 2009.
[12] S. Kulishov and O. Iakovenko, "Individual diagnostic value of monitoring blood glucose and arterial pressure in the patients with diabetes mellitus and essential hypertension: a case report," Final programme \& Abstract Book of the European Conference. IInd edition "Heart, Vessels \& Diabetes", no. 3-5, Athens, Greece, pp. 51, 2011.

[13] G. Seemann, Ep. Scholz, D1. Weiss, and O. Dossel, "Effects of the Reggae Mutations on Synus Node Function: A Simulation Study," Computers in Cardiology, vol. 35, pp. 421-424, 2008.

[14] V. D. Parondzhanov, "Learn to write, read and understand algorithms Algorithms for the correct thinking. Algorithmic Basics," M.: DMK Press, pp. 520, 2012.

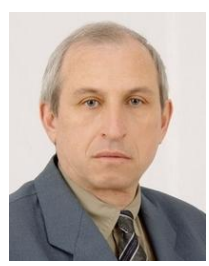

Kulishov Sergii Kostyantynovych is a professor, Ph.D., D. Med. Sci. Higher state educational institution of Ukraine (HSEIU) "Ukrainian Medica Stomatological Academy", Poltava, Ukraine, office address: Kulishov S. K., department of internal medicine No. 1, HSEIU "Ukrainian Medical Stomatological Academy", street Shevchenko, 23 Poltava, Ukraine, 36024; Members of International Association of Computer Science and Information Technology (IACSIT), Acute Cardiac Care, Association(ACCA), the International E-Learning Association (IELA)

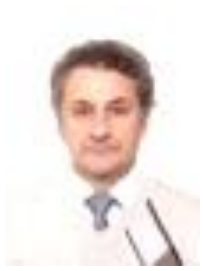

Olexandr Iakovenko is head of endocrinology separation of daily permanent establishment. V. P. Komissarenko Institute of Endocrinology and Metabolism AMS Ukraine, Kyiv, office address: Iakovenko O. M., street Vyshgorodskaia 69, Kyiv, Ukraine, 0414 